\title{
Fabrication and Characterization of Copper Phthalocyanine- Based Field Effect Transistors
}

\author{
Nergui Uranbileg ${ }^{1,2}$, Tuul Tsagaantsooj ${ }^{2}$, Anar Enkhbayar ${ }^{2}$, Davaajargal \\ Darambazar ${ }^{2}$, Munkh-Erdene Erdene-Ochir ${ }^{2}$, Ganzorig Chimed ${ }^{2, *}$ \\ ${ }^{1}$ Institute of Chemistry and Chemical Technology, Mongolian Academy of Sciences, 13330 Ulaanbaatar, \\ Mongolia \\ ${ }^{2}$ Center for Nanoscience and Nanotechnology, Department of Chemical and Biological Engineering, School of \\ Applied Sciences and Engineering, National University of Mongolia, 14201 Ulaanbaatar, Mongolia \\ *Corresponding author. Email: ch_ganzorig@num.edu.mn
}

\begin{abstract}
Future generations of electronic products will be enabled by flexible electronic circuits, displays, and sensors based on organic active materials, which could eventually reach the mainstream electronics industry. One of such devices is the organic field-effect transistor (OFET), which are three-terminal devices that are comprised of a gate, source, and drain electrode. In this study, we fabricated a bottom-gate bottom-contact OFET device using copper phthalocyanine $(\mathrm{CuPc})$ as a semiconducting layer. $\mathrm{CuPc}$ is a commercially available metal complex, a known p-type semiconducting material. Au/Ti electrode is sputtered on Al gated silicon substrate with thermally grown $\mathrm{SiO}_{2}$ dielectric layer. $\mathrm{CuPc}$ films were then deposited over the substrate with patterned electrodes by physical vapor deposition at a rate of $0.35 \mathrm{~nm} / \mathrm{s}$, recorded by a quartz crystal microbalance at room temperature under a background pressure of $1.21 \times 10^{-3} \mathrm{~Pa}$. A thin layer of organic material was also deposited on glass slides and the optical properties of films with different thicknesses were determined by UV-Vis spectrometry and the optical band-gap energy was determined to be $1.64 \pm 0.01 \mathrm{eV}$. The thermal annealing effect on thin-film crystallization morphology was studied with atomic force microscopy (AFM) and contact angle measurement.
\end{abstract}

Keywords: Field-effect transistor, Organic semiconductors, Optical band gap

\section{INTRODUCTION}

Organic materials have several unique properties that are not available in conventional inorganic semiconductors. One of the most promising advantages is that these organic materials can be processed at low temperatures, which makes them suitable for direct processing on flexible plastic substrates [1]. Organic semiconductors are soluble in a variety of solvents at low temperatures so flexible electronic devices can be printed out [2]. Organic field-effect transistor (OFET) mobility has increased greatly over the last three decades, from $10^{-5}$ to over $10 \mathrm{~cm}^{2} / \mathrm{Vs}$ with six orders of magnitude [3]. The semiconductor is either n-type or p-type depending on its dominant carrier type and high mobility is preferred to enable good charge transport and the most widely studied organic semiconductors have been p-type materials [4]. Various structures of OFETs have been used depending on the purposes of the studies. Out of the four possible OFET structures: top-contact bottom-gate (TCBG), bottom-contact bottom gate (BCBG), top-contact top-gate (TCTG), and bottom-contact top-gate (BCTG) structures, the bottom-contact configuration is the more preferred device geometry for manufacturing compared to topcontact structures [5]. This structure, optimize the transistor performance by modifying or altering semiconductor materials required and result in less experimentation for integration. In our study, we used commercial copper phthalocyanine as an organic semiconductor. Figure 1 (a) shows the molecular structure of a p-type $\mathrm{CuPc}$ organic semiconductor molecule, and it has good electrical performance and stability in a transistor configuration and features a non-degenerative feature in ambient air. In the case 
of p-channel OFETs, holes are injected from the gold electrode to the HOMO level of the organic semiconductor, so for effective charge injection, the work function of the electrodes must be closer to the HOMO level of the organic semiconductor material $[5,6]$.

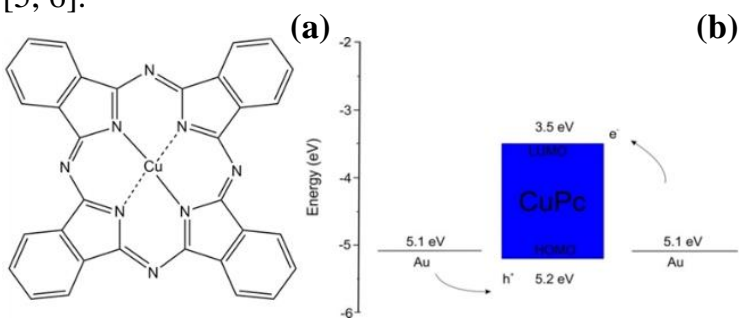

Figure 1. (a) $\mathrm{CuPc}$ molecular structure, (b) Energy diagram of $\mathrm{CuPc}$ with gold source and drain electrodes

The objective of this work is to fabricate organic field-effect transistors through the physical vapor deposition method and study their optical, morphological, and electrical properties. The current work employs a widely used, commercially available, and hole-transporting small molecule semiconductor copper phthalocyanine as p-channel active material on $\mathrm{SiO}_{2}$ dielectric and gold electrodes with a bottomgate bottom-contact structure.

\section{EXPERIMENTAL}

Here, n-type Si wafers $<100>(0.0029 \Omega \cdot \mathrm{cm})$ with thermally oxidized $300 \mathrm{~nm} \mathrm{SiO}_{2}$ dielectric are used as a substrate. The wafers were cleaned by the RCA standard cleaning process. Then, $100 \mathrm{~nm}$ thick aluminum was deposited onto the back of the $\mathrm{Si}$ substrate by thermal evaporation as a back gate. Next, $130 \mathrm{~nm}$ gold source-drain electrodes were sputtered on the $\mathrm{SiO}_{2}$ through the use of a shadow mask ( $W=5000 \mathrm{~mm}, L=1000 \mathrm{~mm}$ ). Finally, CuPc films were thermally evaporated on devices and glass slides to various thicknesses at a rate of $0.08-0.13$ $\mathrm{nm} / \mathrm{s}$, as recorded by a quartz crystal microbalance (INFICON SQM-160) under a background pressure of $1.05 \times 10^{-3} \mathrm{~Pa}$. The schematic representation and images of fabricated devices are shown in Figure 2.

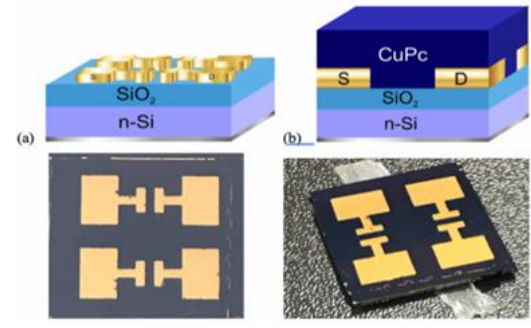

Figure 2. Before and after depositing $\mathrm{CuPc}$ of (a) 3D models and (b) real images
The optical properties of $\mathrm{CuPc}$ thin film deposited on glass slides are measured in the range of $280 \mathrm{~nm}$ to $800 \mathrm{~nm}$ at room temperature by Shimadzu UV2500PC spectrophotometer. The water contact angle (WCA) was measured through a sessile drop approach using a motorized drop-shape analysis system (DSA100, KRÜSS). All measurements were taken at ambient temperature. The surface morphology of thin films and devices were studied by atomic force microscope (AFM-SPI 3700) in contact mode in ambient atmosphere. Current-voltage (I-V) measurements were carried out at room temperature using a Keithley 236 source measure unit and Advantest R6145 as an external DC source.

\section{RESULTS AND DISCUSSION}

The UV-Vis spectrum for phthalocyanine originates from molecular orbitals within the aromatic $18 \mathrm{p}$ electron system and form overlapping orbitals on the central metal. The conjugated double bonds within the crystal structure of the film create electron orbitals that overlap between molecular $p$ orbitals. These electrons can transfer energy throughout the structure and are responsible for the absorption peaks. Since the absorption spectrum reveals information on electronic transition, the onset of absorption can be considered as the band gap of the compound [7]. The optical absorption spectrum of $\mathrm{CuPc}$ with different thicknesses is shown in Figure 3 and 4. In the near UV region, a single peak is observed around $330 \mathrm{~nm}$ correspond to the Soret $(B$ band) related to free-electron oscillation inside the copper atoms. A doublet of peaks existing in the visible region around $616 \mathrm{~nm}$ and $694 \mathrm{~nm}$ are known as the Q-band and have generally been interpreted in terms of $\pi-\pi^{*}$ electron transition from the valence band to the conduction band of phthalocyanine. It can also be noticed that this band shows the characteristic splitting (Davydov splitting) present in all phthalocyanine derivatives. The high-energy peak of the Q-band has been assigned to the first $\pi-\pi^{*}$ transition on the phthalocyanine macrocycle. The UV-Vis spectra show characteristic features of $\alpha$ phase $\mathrm{CuPc}$ because in Q-band the lower energy peak is weaker than that of the high energy peak [8]. Figure 3 shows the intensity of absorption peaks in $\mathrm{CuPc}$ depend on the thickness of the film. 


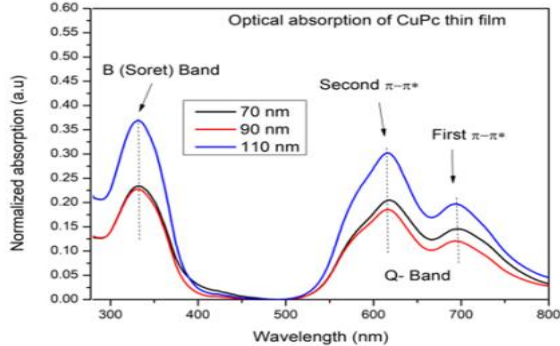

Figure 3. Absorption spectrum of different thicknesses of $\mathrm{CuPc}$ on a glass slide

The Tauc plot in Figure 4 shows the determination of the band gap from the absorption spectrum of CuPc. The optical band gap energy is about $1.64 \pm 0.01 \mathrm{eV}$ and its in range of previously reported band gap of $\mathrm{CuPc}$ thin films [9].

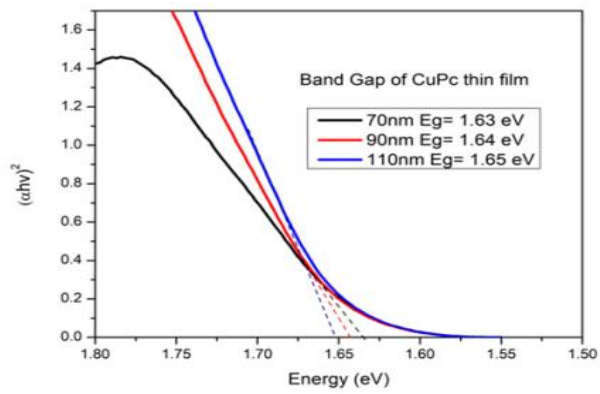

Figure 4. Calculated band gaps of CuPc thin films with different thicknesses

Furthermore, morphologies of the thin films were characterized by AFM. Figure 5-7 shows the topographical images of the CuPc films grown on a glass substrate with various thicknesses $(70,90$, and $110 \mathrm{~nm}$ ) in a scanning area of $2.5 \times 2.5 \mu \mathrm{m}$. The coalescence of grains leads to an increase in grain size that has the lowest surface energy, resulting in improved crystallinity and fewer grain boundaries allowing easy charge hopping between the molecules. It is accepted that the surface roughness and the grain size are important parameters that affect the electrical performance of OFETs.

(a)
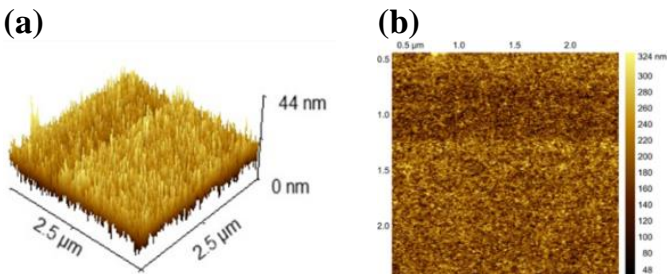

Figure 5. AFM image (a) 3D (b) top-view of $70 \mathrm{~nm}$ $\mathrm{CuPc}$ deposited on a glass substrate (a)

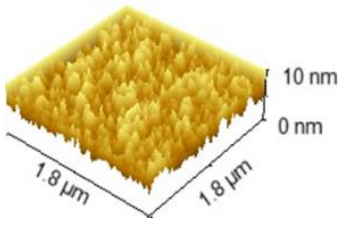

(b)

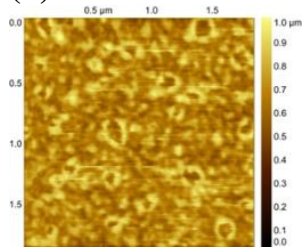

Figure 6. AFM image (a) 3D (b) top-view of $90 \mathrm{~nm}$ $\mathrm{CuPc}$ deposited on a glass substrate

As seen in Figure 5, the $70 \mathrm{~nm} \mathrm{CuPc}$ thin film has homogenous small crystal grains with an average diameter of $186 \mathrm{~nm}$. The roughness for the $\mathrm{CuPc}$ thin film was found to be $44 \mathrm{~nm}$.

To get a clearer AFM image, the original scanned area of $90 \mathrm{~nm}$ and $110 \mathrm{~nm} \mathrm{CuPc}$ films, has been decreased to $1.8 \times 1.8 \mu \mathrm{m}$. As shown in Figure 6 , the morphology of $90 \mathrm{~nm} \mathrm{CuPc}$ film shows homogenous small crystal grains with an average diameter of 684 $\mathrm{nm}$. On the other hand, the $110 \mathrm{~nm} \mathrm{CuPc}$ film show homogenous small crystal grains with an average diameter of $256 \mathrm{~nm}$ in Figure 7. (a)

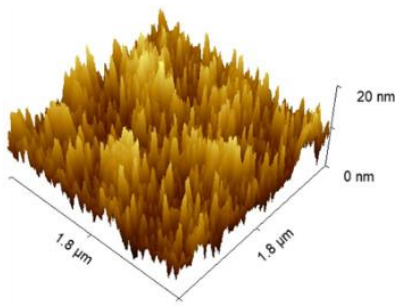

(b)

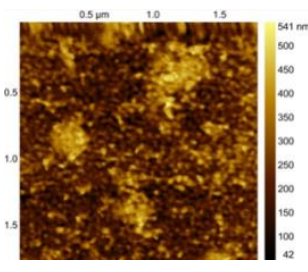

Figure 7. AFM image (a) 3D (b) top-view of $110 \mathrm{~nm}$ CuPc deposited on a glass substrate

In Table 1, RMS roughness and average values of different thicknesses of $\mathrm{CuPc}$ on glass substrate are written.

Table 1. Surface roughness and average value for each film

\begin{tabular}{|l|l|l|}
\hline Thickness & $\begin{array}{l}\text { RMS Roughness } \\
(\text { Sq })\end{array}$ & $\begin{array}{l}\text { Average grain } \\
\text { size }\end{array}$ \\
\hline $70 \mathrm{~nm}$ & $44.81 \mathrm{~nm}$ & $186.8 \mathrm{~nm}$ \\
\hline $90 \mathrm{~nm}$ & $100.8 \mathrm{~nm}$ & $684.4 \mathrm{~nm}$ \\
\hline $110 \mathrm{~nm}$ & $80.3 \mathrm{~nm}$ & $265.8 \mathrm{~nm}$ \\
\hline
\end{tabular}

In Figure 8, the water contact angle was measured of different thicknesses on the glass substrate. The obtained WCAs were determined from an average of left and right contact angles. To enhance the testing precision, the deionized water droplet $(5 \mu \mathrm{L})$ was deposited onto the surface of the treated samples. A snapshot of the drop shape is captured $10 \mathrm{~s}$ after drop deposition, which is beneficial for the equilibrium 
establishment of the solid-liquid-gas interface. The increased surface roughness typically leads to lower surface energy which would result in the hydrophobic surface; thus the difference in surface roughness would lead to a change in water drop contact angle [10]. In Figure $8 \mathrm{~b}$ and $\mathrm{c}$, the $\mathrm{CuPc}$ films are shown to be hydrophilic but it is likely due to the dissolution of the film at the water-film interface.

The $\alpha$ and $\beta$ forms the most frequently encountered states of $\mathrm{CuPc}$. The $\alpha-\mathrm{CuPc}$ form is metastable at $\mathrm{T}=165^{\circ} \mathrm{C}$ and can be converted thermally or with a solution to be $\beta$ form. The conductivity of $\alpha-\mathrm{CuPc}$ is higher than that of $\beta-\mathrm{CuPc}$, due to the higher $\pi-\pi$ stacking in $\alpha$ form [8].
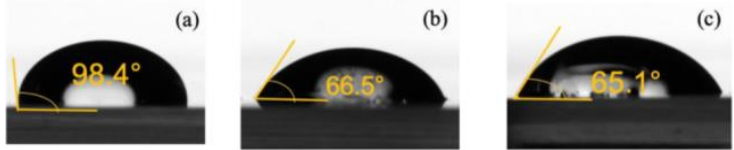

Figure 8. Contact angle of (a) $70 \mathrm{~nm}$ (b) $90 \mathrm{~nm}$ (c) $110 \mathrm{~nm}$ thicknesses of $\mathrm{CuPc}$ on a glass substrate

The annealing process is a broadly employed method to enhance the quality of the crystal and exploit structural defects in the material. To that effect, annealing of the prepared films was carried out at different temperatures in $100^{\circ} \mathrm{C}, 165^{\circ} \mathrm{C}, 200^{\circ} \mathrm{C}$ for two hours. Figure 9 shows the absorption spectra of annealed films at three different temperatures and the intensity of absorption peaks decreases as the annealing temperature increases.
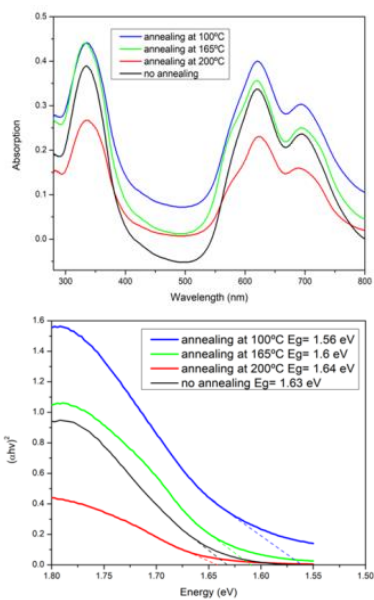

Figure 9. Absorption spectrum of annealed devices spectrum of annealed devices and band gap energies of annealed films

The calculated band gaps of the prepared and film annealed at $200^{\circ} \mathrm{C}$ are similar in range with 1.63 and $1.64 \mathrm{eV}$ respectively. However, the band gap of the films annealed at $165^{\circ} \mathrm{C}$ and $200^{\circ} \mathrm{C}$ show some unexpected values and this is probably due to measurement error. Thus, the obtained results overall are not enough to draw a meaningful conclusion about the relationship between the band gap value and annealing temperatures. During thermal annealing, if the crystallization of organic molecules is affected then there may be a noticeable change regarding the morphology and structural properties of the resulting film. (a)

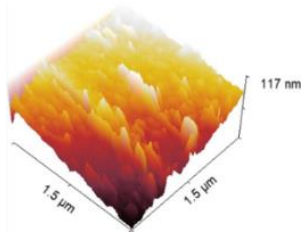

(b)

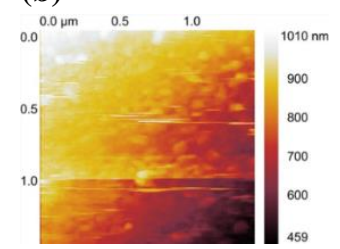

Figure 10. AFM image (a) 3D (b) top-view of $80 \mathrm{~nm}$ deposited glass substrate annealing at $100^{\circ} \mathrm{C}$

Additionally, the surface morphology of the semiconducting layer plays a crucial role in defining the device performance, so the morphological analysis is conducted on $80 \mathrm{~nm} \mathrm{CuPc}$ film annealed at temperatures of $100^{\circ} \mathrm{C}, 165^{\circ} \mathrm{C}$, and $200^{\circ} \mathrm{C}$. From the AFM images, it is seen that the roughness and grain size of CuPc films decrease with temperature.

The morphology of the annealed CuPc thin film has a large grain size and is homogeneously distributed, which indicates the crystalline nature of the film. It is obvious because the films were prepared at different annealing temperatures and due to the heating, grain growth will take place, which will affect the crystallinity of the film. Figure 10 shows a uniform distribution of a granular grain structure which is consistent with a high-quality thinfilm nanostructure with a pin-hole-free, smooth, and crack-free surface. (a)

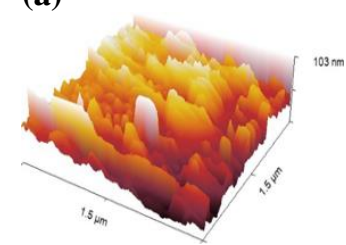

(b)

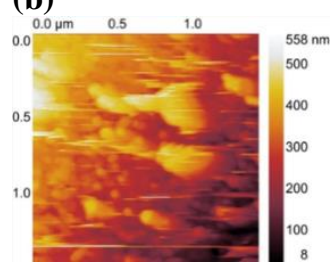

Figure 11. AFM image (a) 3D (b) top-view of $80 \mathrm{~nm}$ deposited glass substrate annealing at $165^{\circ} \mathrm{C}$

Surface of the films consisted of many granular crystallites with regular sizes, and the crystallite size increased as the temperature is increased as seen in Figures 11 and 12. Surface roughness and the average value for each annealed film are summarized in Table 4. 
(a)

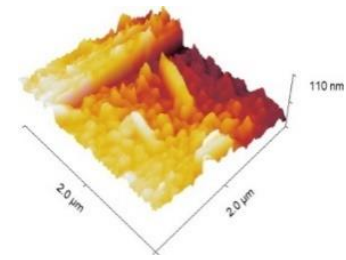

(b)

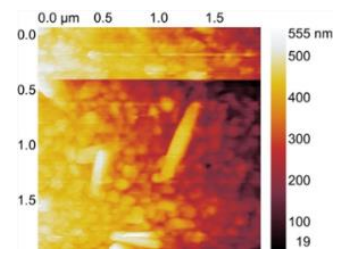

Figure 12. AFM image (a) 3D (b) top-view of $80 \mathrm{~nm}$ deposited glass substrate annealing at $200^{\circ} \mathrm{C}$

Table 2. Surface roughness and average value for each film

\begin{tabular}{|l|l|l|}
\hline $\begin{array}{l}\text { Annealing } \\
\text { temperature }\end{array}$ & RMS roughness & Average value \\
\hline $100^{\circ} \mathrm{C}$ & $117.7 \mathrm{~nm}$ & $787 \mathrm{~nm}$ \\
\hline $165^{\circ} \mathrm{C}$ & $103.3 \mathrm{~nm}$ & $284.3 \mathrm{~nm}$ \\
\hline $200^{\circ} \mathrm{C}$ & $110.8 \mathrm{~nm}$ & $324.4 \mathrm{~nm}$ \\
\hline
\end{tabular}

In Figure 13, measured water contact angles of $\mathrm{CuPc}$ films after thermal annealing. As seen from the previous result in Figure 8 the films show hydrophilic characteristics but the same dissolution at the interface is likely the cause rather than the annealing effect.

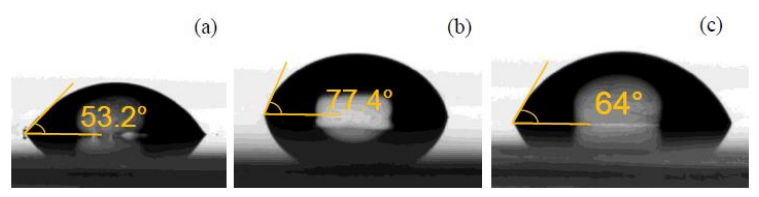

Figure 13. Contact angle of $\mathrm{CuPc}$ on glass substrate annealed at (a) $100^{\circ} \mathrm{C}$ (b) $165^{\circ} \mathrm{C}$ (c) $200^{\circ} \mathrm{C}$ temperature

In the case of electrical characterization, the typical electrical characteristics of OFET devices are obtained in form of output and transfer curves of the devices. The output characteristic curve is obtained by a applying voltage between the source and drain electrode when the applied gate voltage is kept constant while the transfer characteristics are obtained by measuring $I_{D S}$ while sweeping the $V_{G}$ when $V_{D S}$ is kept constant [11]. First, the $I_{D S}$ is swept from $-10 \mathrm{~V}$ to $10 \mathrm{~V}$ in steps of $0.5 \mathrm{~V}$ and $I_{D S}$ is measured when $V_{G}$ is $0 \mathrm{~V}$. Then the gate voltage was increased to $V_{G}=-1 \mathrm{~V}$. The source-drain voltage and current are both sourced measured by and the Keithley 236 source measure unit and the gate voltage are supplied by external DC power source Advantest R6145. The results are shown in Figure 14. The results of the electrical measurement were unfortunately unsuccessful in our attempt to measure our CuPc OFET devices. When the drain current was measured without any applied voltage at the gate electrode the amount of current measured was significantly high and when the $-1 \mathrm{~V}$ voltage was applied at the gate electrode and the drain current was measured, a visible spark went off between the gold electrodes and the measured current reached the preset $10 \mathrm{~mA}$ compliance limit leading to a device breakdown.

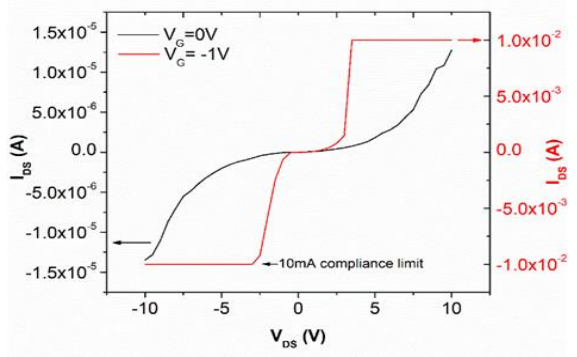

Figure 14. Measured $I_{D S}$ current as $V_{D S}$ is swept from $-10 \mathrm{~V}$ to $10 \mathrm{~V}$ with $\mathrm{V}_{\mathrm{G}}=0$ (black) and $\mathrm{V}_{\mathrm{G}}=-1 \mathrm{~V}$ (red)

Furthermore, even without applying any potential to the gate electrode after one or two $I_{D S}$ measurements with $V_{D S}$ in the range of $-10 \mathrm{~V}$ to $10 \mathrm{~V}$ same breakdown was observed. The breakdown of devices at such low operating voltages has prevented us from obtaining any useful results.

\section{CONCLUSION}

The optical absorption measurements of the CuPc films show the characteristic absorption profile as described in previous studies. The optically determined band gap of the $\mathrm{CuPc}$ is close to the energy difference between HOMO and LUMO level of the CuPc. The annealing effect on the optical absorption showed no significant difference. Thermal annealing typically improves the molecular order and result in the increased crystalline film. Thus, we tried to characterize that effect by measuring surface morphology and energy by AFM and contact angle measurement. Although the samples show hydrophilic properties, it was attributed to the dissolution of the film which interfered with the measurement. From AFM measurement, the roughness and grain size of CuPc films decreases with temperature. Small grain size decreases the charge transport properties and higher surface roughness results in the large numbers of trap formations which reduce the transistor performance. 


\section{ACKNOWLEDGMENTS}

This work has been done within the funding of the School of Applied Sciences and Engineering, National University of Mongolia.

\section{REFERENCES}

[1] Sazonov A., Striakhilev D., Lee C.H., Nathan A., (2005) Low-temperature materials and thin film transistors for flexible electronics. Proceedings of the IEEE, Vol. 93(8), 1420-1428.

[2] Zhang Z., Qiu J., Wang S., (2016) Roll-to-roll printing of flexible thin-film organic thermoelectric devices. Manufacturing Letters, Vol. 8, 6-10.

[3] Paterson A.F., Singh S., Fallon K.J., Hodsden T., Han Y., Schroeder B.C., et al., (2018) Recent progress in high-mobility organic transistors: a reality check. Advanced Materials, Vol. 30(36), 1801079.

[4] Yamashita Y., (2009) Organic semiconductors for organic field-effect transistors. Sci. Technol. Adv. Mater, Vol. 10(2), 024313.

[5] Lamport Z.A., Haneef H.F., Anand S., Waldrip M., Jurchescu O.D., (2018) Tutorial: Organic field-effect transistors: Materials, structure and operation. Journal of Applied Physics, Vol. 124(7), 071101.

[6] Siol C., Melzer C., Seggern H., (2008) Electron trapping in pentacene based $\mathrm{p}$ - and n-type organic field-effect transistors, Applied Physics Letters, Vol. 93(13), 133303.

[7] Gómez A.R., Sánchez-Hernández C.M., Fleitman-Levin I., Arenas-Alatorre J., AlonsoHuitrón J.C., Vergara M.E.S., (2014) Optical absorption and visible photoluminescence from thin films of silicon phthalocyanine derivatives. Materials, Vol. 7(9), 6585-6603.

[8] Zou T., Wang X., Ju H., Zhao L., Guo T., Wu W., Wang H., (2018) Controllable molecular packing motif and overlap type in organic nanomaterials for advanced optical properties. Crystals, Vol. 8(1), 22.

[9] Mali S.S., Dalavi D.S., Bhosale P.N., Betty C.A., Chauhan A.K., Patil P. S., (2012) Electrooptical properties of copper phthalocyanines $(\mathrm{CuPc})$ vacuum deposited thin films. RSC advances, Vol. 2(5), 2100-2104.
[10] Ubuo E.E., Udoetok I.A., Tyowua A.T., Ekwere I.O., Al-Shehri H.S., (2021) The Direct Cause of Amplified Wettability: Roughness or Surface Chemistry? Journal of Composites Science, Vol. 5(8), 213.

[11] Choi D., Chu P. H., McBride M., Reichmanis E., (2015) Best practices for reporting organic field effect transistor device performance. Chemistry of Materials, Vol. 27(12), 41674168. 